\title{
Comparative Analysis of the GIS-based Trophic States of Lakes and Reservoirs in Shandong Province
}

\author{
Baohua Zhou ${ }^{1, \text { a }}$ and Zhanhong Liu $^{1, b^{*}}$ \\ ${ }^{1}$ Resources and Environmental Institute, University of Jinan, Jinan, China, 250022 \\ ${ }^{a}$ stu_zhoubh@ujn.edu.cn, bstu_liuzh@ujn.edu.cn \\ *The corresponding author
}

Keywords: Lakes and reservoirs in Shandong; Trophic state; Comparison of lakes and reservoirs

\begin{abstract}
According to the relationship between water quality parameters and trophic state index, as well as on the basis of the dividing lakes and reservoirs into four regions, the regional differences of water quality parameters is analyzed by using ArcGIS software, and the parameters include total nitrogen concentration, total phosphorus concentration, chlorophyll a concentration and Secchi disk depth. Then the trophic states of Laoshan Reservoir and Jihongtan reservoir in same region, Nansi Lake and Dongping Lake in different regions were compared and analyzed respectively. The results show that: the trophic states of lakes and reservoirs in the mountainous are better than that of those in the plain; the pollution situation is more serious and the trophic states are worse in industrial areas and populated area.
\end{abstract}

\section{Introduction}

Lakes and reservoirs are indispensable members of nature, important carriers of freshwater resources, which not only carry the function to provide water resources, develop tourism and storage flood, but also the special functions of monitoring regional climate and maintaining regional ecological balance[1]. However, due to long terms of irrational development and utilization by human, water quality problems of lakes and reservoirs have becoming increasingly severe in recent years and eutrophication has become one of the most serious water quality problems in the world. In the early 20th century, eutrophication problems of water had already aroused the attention of ecologists and limnologists, scholars all over the world began to conduct extensive and in-depth research in this area. In the late 1960s, UNFAO(United Nations Food and Agriculture Organization), UNESCO(United Nations Educational, Scientific and Cultural Organization), EEC(European Economic Community), WHO(World Health Organization) and many other international organizations set up specialized research studies on water eutrophication problem and has obtained numerous research findings[2]. From the late 1950s till mid 1980s, China had successively carried out systematic comprehensive or thematic survey on the nation's major lakes which laid the foundation for research on lakes and reservoirs[3].

In Shandong, there is a large number of lakes and reservoirs, with lake area of $1496.6 \mathrm{~km} 2$ and perennial storage capacity of $23.53 \times 108 \mathrm{~m} 3$ mainly located on the contact zone between the province's Middle Southern mountainous area and West plain. These lakes and reservoirs are divided into two lake groups - Nansi Lakes and Beiwu Lakes - with Jining as the center[4]. Currently, there are 5589 reservoirs in Shandong with a total storage capacity of approximately $155.06 \times 108 \mathrm{~m} 3$. Among which, there are 32 large reservoirs, 152 medium sized reservoirs and 5405 small reservoirs[5].

By collecting data of lakes and reservoirs in Shandong Province and dividing them according to different geographical regions, this study carried out comparative analysis on different lakes and reservoirs on the bases of four parameters which include total nitrogen, total phosphorus, chlorophyll a and Secchi disk depth, and proceeded on to make comprehensive evaluations which provides basis for managing lakes and reservoirs in Shandong. 


\section{Division of Lakes and Reservoirs}

Division of lakes and reservoirs mainly follows the principle of consistency of supply source of the lakes and reservoirs. The consistency of supply source of lakes and reservoirs depends on geological and geomorphologic factors. Water features of lakes and reservoirs with different climatic characteristics also cause differences. Therefore, dividing regions of lakes and reservoirs in Shandong Province mainly selected factors such as topography, climate, water supply and administrative divisions etc, to carry out comprehensive analysis. With reference to existing water resource division of the province river basin, we divided the lakes and reservoirs in Shandong Province into four divisions to study: Haihe River Basin Lakes and Reservoirs Area (A), Yellow River Basin Lakes and Reservoirs Area (B), Yishusi River Lakes and Reservoirs Area (C) and Eastern Shandong Peninsula Lakes and Reservoirs Area (D), Figure 2.1 is made under GIS.

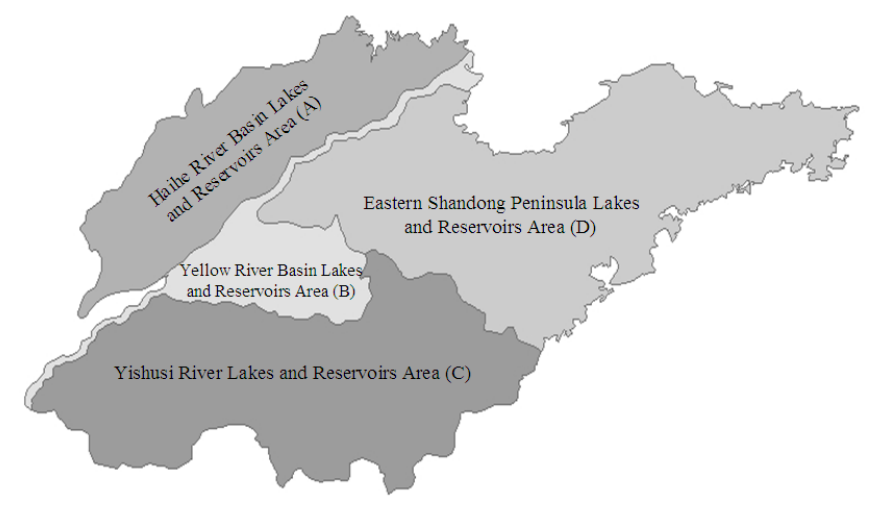

Figure 1. Division of lakes and reservoirs in Shandong Province

\section{Divisional Evaluation of Trophic States of Lakes and Reservoirs}

Eutrophication is a phenomenon when the double impact of natural environmental factors and human activities cause large input of nutrients into water, which lead to gradual change of the water from an oligotrophic state of low productivity level to a eutrophic state. It is also an indication of the degree of aging of trophic state during lakes and reservoirs evolution process.

Choose Evaluation Factors. The trophic state of lakes and reservoirs mainly depends on the accumulation level of nutrients and organic matters in the lakes and reservoirs. In China, we generally selects chlorophyll a ( Chl-a ) concentration, total nitrogen ( TN ) concentration, total phosphorus ( TP ) concentration, potassium permanganate index $\left(\mathrm{COD}_{\mathrm{Mn}}\right)$ and Secchi disk depth (SD) which can directly reflect trophic states of lakes and reservoirs as the five basic parameters to evaluate trophic states of lakes and reservoirs. Among them, Chl-a concentration can directly reflect the concentration of algae, thus known as "elementary factors", TN concentration and TP concentration are the main limiting factors for algae proliferation, thus are known as the "critical factors" ${ }^{[6]}$. In this study, the collection of permanganate index $\left(\operatorname{COD}_{\mathrm{Mn}}\right.$ in ), total nitrogen (TN) concentration data of the selected lakes and reservoirs is incomplete, thus TN concentration date is replaced by ammonia nitrogen ( $\mathrm{NH} 3-\mathrm{N}$ ) concentration data as the measuring parameter. Therefore, our research mainly used chlorophyll a ( Chl-a ) concentration, ammonia ( NH3 -N ) concentration, total phosphorus ( TP) concentration, Secchi disk depth (SD) which can directly reflect trophic states of lakes and reservoirs as four basic parameters for the evaluation of trophic state of lakes and reservoirs in Shandong.

The Spatial Interpolation Method of the GIS to Seek Mean Value of Division Index. The spatial interpolation method is a calculation method in GIS which uses data of known points to deduce data of unknown points in the same region. It is commonly used to convert discrete points of measured date into continuous surface of data, so as to compare with the distribution pattern of phenomena of other space. 
This study uses data provided by National Drinking Water Source Investigation Program, Jinan Environmental Protection Bureau, Shandong Monitoring Station, and Jinan Environment Bulletin, etc, and selected relevant data collected in 2010 of lakes and reservoirs in Shandong for interpolation analysis. The selected lakes and reservoirs mainly include: Xiashan Reservoir of Weifang, Menlou Reservoir of Yantai, Gaoling Reservoir, Laoshan Reservoir of Qingdao, Jihongtan Reservoir of Qingdao, Wohushan Reservoir, Jinxiuchuan Reservoir, Nansi Lake, Dongping Lake, Mata Lake and Daming Lake. Due to financial and resource constrains, there are limitations in monitoring of lakes and reservoirs, thus lakes and reservoirs selected in this study are not comprehensive enough. However, to some extent, they are able to reflect the trophic states of lakes and reservoirs in their areas. On the bases of selected lakes and reservoirs, we used ArcGIS software to process the data from lakes and reservoirs, applied spatial interpolation method for statistical analysis of the four lakes and reservoirs area, and obtained the means of four basic water quality indicators - NH3-N, TP, Chl-a and SD - in each region.

Choose Evaluation Method. The evaluation of the trophic states of lakes and reservoirs uses a series of relevant indicators as well as their mutual relationships to make accurate judgments on the trophic states of lakes and reservoirs[7]. Current methods to evaluate the trophic states of lakes include: trophic states index, trophic level index and Carlson trophic states index. This study applied the comprehensive trophic states index $\operatorname{TLI}(\Sigma)$ to process and compute relevant data and draw out the nutrition state index with respect to water quality, therefore evaluate the trophic level of the water body.

Equation selected to calculate trophic states index:

$$
\boldsymbol{T L I}\left(\sum\right)=\sum_{j=1}^{m} W \boldsymbol{j} \bullet T L I(j)
$$

Where: $\operatorname{TLI}\left(\sum\right)$-- comprehensive nutrition states index

$\mathrm{W}_{\mathrm{j}}$-- weight of the $\mathrm{j}$ parameter of trophic states index

TLI(j) -- the trophic states index representing the $\mathrm{j}$ parameter

TLI(j) equation for some parameters of trophic states index:

$\mathrm{TLI}(\mathrm{Chl}-\mathrm{a})=10(2.5+1.086 \mathrm{LnChla})$;

$\mathrm{TLI}(\mathrm{TP})=10(9.436+1.624 \mathrm{LnTP})$;

$\operatorname{TLI}\left(\mathrm{NH}_{3}-\mathrm{N}\right)=10\left(5.453+1.694 \mathrm{Ln} \mathrm{NH}_{3}-\mathrm{N}\right)$;

$\mathrm{TLI}(\mathrm{SD})=10(5.118-1.94 \mathrm{LnSD})$

Where: the unit for Chlorophyll a is $\mathrm{mg} / \mathrm{m}^{3}$, the unit for Secchi disk depth is $\mathrm{m}$, units for other parameters are $\mathrm{mg} / \mathrm{L}$.

In order to determine the stage of development of the lakes and reservoirs in the process of eutrophication, a series of consecutive numbers 0 - 100 are chosen to grade the trophic states of the lakes and reservoirs and are divided into three trophic levels - poor, medium and rich (Table 1). At same trophic state, higher index value indicates a more severe trophic degree.

Table 1 Relation between trophic level and trophic states

\begin{tabular}{|c|c|c|c|}
\hline $\begin{array}{c}\text { Comprehensive } \\
\text { nutrition states index }\end{array}$ & poor & $\begin{array}{c}\text { mediu } \\
\mathrm{m}\end{array}$ & rich \\
\hline $\mathrm{TLI}\left(\sum\right)$ & $\leq 30$ & $\leq 50$ & $>50$ \\
\hline
\end{tabular}

Evaluation Result. We applied comprehensive nutrition states index method on the mean value of the four basic water quality indicators - NH3-N, TP, Chl-a , and SD - within each region, and calculated the trophic states index and comprehensive nutrition index of the four indicator. The final evaluation result of trophic states is shown in Table 2. As we did not select lakes and reservoirs from the Haihe River Basin lakes and reservoirs, due to scientific considerations, we will no longer carry out discussions on this in later parts of this paper. 
Table 2 Evaluation result on the trophic states of different regions in 2010

\begin{tabular}{|c|c|c|c|c|c|c|}
\hline Lakes and reservoirs area & $\begin{array}{c}\mathrm{NH}_{3^{-}} \\
\mathrm{N}\end{array}$ & $\mathrm{TP}$ & Chl-a & $\mathrm{SD}$ & $\mathrm{TLI}($ \\
$\left.\sum\right)$ & $\begin{array}{c}\text { Trophic } \\
\text { states level }\end{array}$ \\
\hline Yellow River Basin Lakes & 24.76 & 54. & 51.74 & 48.6 & 45.68 & medium \\
and Reservoirs area & & 17 & & 0 & & \\
\hline Yishusi River Lakes and & 20.91 & 46. & 37.76 & 57.3 & 40.40 & medium \\
Reservoirs area & & 16 & & 7 & & \\
\hline East Shandong Peninsula & 21.34 & 55. & 44.62 & 45.2 & 42.12 & medium \\
Lakes and Reservoirs area & & 36 & & 1 & & \\
\hline
\end{tabular}

\section{Comparison of the Trophic States of Lakes and Reservoirs}

Due to differences in recharging and discharging states of lakes and reservoirs, hydrodynamic conditions within the lakes and reservoirs as well as geological and topographical conditions are different, trophic states of different lake regions will show differences, lakes and reservoirs in same lake region will also have differences in trophic states level. Therefore, on the basis of evaluating trophic states according to division of lakes and reservoirs, we carried out further comparative analysis on the trophic states of lakes and reservoirs in Shandong.

Comparison of Trophic States of Three Lakes and Reservoirs Divisions. From Table 2 we can deduce that: Yishusi River Lakes and Reservoirs area in Shandong has the lowest trophic states, followed by East Shandong Peninsula Lakes and Reservoirs area, Yellow River Basin Lakes and Reservoirs area has a relatively high trophic level, close to an eutrophication level.

Ammonia nitrogen, total phosphorus, chlorophyll a trophic states indexes are the lowest in the three regions for Yishusi River Lakes and Reservoirs area, all laid in medium trophic level, water quality is relatively good. However, the Secchi Disk Depth index is quite high in this area, and has reached a eutrophic level. Total phosphorus and chlorophyll a indexes in the Yellow River Basin Lakes and Reservoirs area all reached eutrophic level, water quality is relatively bad. East Shandong Peninsula Lake Reservoir showed relatively low ammonia concentration, chlorophyll a concentration and Secchi Disk Depth indexes, but highest total phosphorus index, therefore water quality is normal.

The evaluation result in terms of lakes and reservoirs water quality according to regional differences shares commonality with the result in 2010 Environment Bulletin of Shandong Province. This is because there are hydraulic connections between lakes and reservoirs as well as rivers and surrounding basin[8]. Water sources for lakes and reservoirs mainly comes from surface water, such as rivers of upper reaches and nearby seas, in addition part of it also comes from underground water recharge. In rainy seasons, lakes and reservoirs can accept more recharge from river water; in dry seasons, they can recharge surrounding basin through underground runoff. Thus, water quality of lakes and reservoirs largely depends on the water quality of surrounding rivers.

Comparison of Typical Lakes and Reservoirs Trophic States within Same Region. We chose Qingdao Laoshan Reservoir of the East Shandong Peninsula Lake Reservoir area and Jihongtan Reservoir for comparative analysis. Laoshan Reservoir belongs to mountainous lakes and reservoirs area and Jihongtan Reservoir belongs to plain farmland lakes and reservoirs area, thus both have strong representation in terms of geological and geomorphologic differences.

Laoshan Reservoir of Qingdao. Laoshan Reservoir locates in the Laoshan beauty spot. It is one of Qingdao's centralized drinking water sources in the city. The reservoir was built in 1958, surrounded by mountains which form a pelvic floor. Total capacity of the reservoir is $5601 \times 10^{4} \mathrm{~m}^{3}$. The reservoir originates in northern part of Laoshan Mountains. Recharging water for the reservoir mainly comes from Wuyixiang River, Wulong River and Shanseyu River, with a total recharging area of about $99.6 \mathrm{~km}^{2}[9]$. However, with the expanding of city-size in Laoshan district, rapid economic development as well as tourism development, there has been an increase of trophic materials input into Laoshan Reservoir. As a result, the water body has experienced some extent of 
pollution. According to 2009 and 2010 data, trophic states index of Laoshan Reservoir was 48.6 in 2009, which indicates a medium trophic level, trophic states index in 2010 was 52.11, which had reached a light level of eutrophication.

Laoshan Reservoir belongs to mountainous area reservoir with relatively few sources of trophic materials and relatively poor aquatic organism type and quantity. The oxygen dissolving level is adequate, therefore water quality can be considered as good. Statistics show that from 2009 to 2010, ammonia nitrogen concentration, total phosphorus concentration, potassium permanganate indexes are relatively low in Laoshan Reservoir, all on medium trophic level, but chlorophyll a content is considerably high and Secchi Dish Depth is low (less than 0.5m).

Nitrogen, phosphorus and other trophic materials in Laoshan Reservoir mainly come from domestic sewerage and waste water from agriculture activities. Farmland in Laoshan Reservoir area mainly distribute in the reservoir's entrance area and upper reaches of Wulong River, coupled with upper stream's rapid development in fruit industry in recent years and high application rate of pesticides and fertilizer, trophic materials such as nitrogen and phosphorus in soil flow into the reservoir due to rainfall or irrigation. Garbage dumped on the river sides, wastes from livestock manures of the upper stream as well as industrial development of certain industries all caused a certain amount of pollution to the reservoir. In addition, Laoshan Reservoir is a tourist destination. Rapid development in surrounding catering industry and increase in population density both produces large quantity of waste water and garbage, which directly affects the water quality in the reservoir area. Although human activities on the reservoir area caused a certain amount of pollution, with an annual rainfall of $800 \mathrm{~mm}$ in the Qingdao region which not only dilute the concentration of trophic materials and organic matters in the reservoir but also improved the exchange capacity of the water in the lakes and reservoirs. As a result, there is a reduction in pollution level.

Chlorophyll a concentration is an indication of the types and quantity of phytoplankton and algae species in the water. Its influencing factors are multi-facial. Nutrient salts (nitrogen, phosphorus), precipitation, light and water dynamics been considered as the key affecting factors[10-11]. Laoshan Reservoir is located in coastal area with suitable light and temperature condition, a large quantity of algae and phytoplankton multiply rapidly and increase the chlorophyll a concentration in the reservoir area.

Factors affect the Secchi Disk Depth of water includes: physical and chemical properties of water, solar radiation, composition and concentration of suspending materials as well as meteorological conditions, among them, composition and concentration of suspending materials is the main factor[12]. The Chlorophyll a concentration in Laoshan Reservoir is relatively high. This, to some extent, lowered the Secchi Dish Depth of the lakes and reservoirs. Furthermore, wind speed in coastal areas is high, sediments under water bed of the lakes and reservoirs can suspend easily under disturbance which increased the concentration of suspending materials in the water and lowered the Secchi Dish Depth of the water.

Jihongtan Reservoir of Qingdao. Jihongtan Reservoir is the only reservoir involved in the Water Diversion Project from Yellow River to Qingdao in Shandong. It is located on the junction of Jiaozhou City, Jimo City and Chengyang District. The reservoir is the largest plain dam-type reservoir with dam length of $14.2 \mathrm{~km}$ and total capacity of $1.568 \times 10^{8} \mathrm{~m}^{3}$, supplying over $50 \%$ of residential water usage for the urban area of Qingdao[13].

According to data collect from 2005 to 2010, ammonia concentration, Secchi Dish Depth and Chlorophyll a concentration in Jihongtan Reservoir are all at medium trophic level. Average Secchi Disk Depth was $1.7 \mathrm{~m}$. Potassium permanganate index in 2006 and 2010 are at medium trophic level with that of other years been at poor level. Phosphorus concentration was the best, been at poor trophic level all the years. Compare with Laoshan Reservoir, water quality in Jihongtan Reservoir is relatively good, in 2009 trophic states index was 39.5 and in 2010 trophic states index was40.9, overall water quality was in medium trophic level.

Jihongtan Reservoir is a dam type plain reservoir with closed circumference, thus collects reflectively smaller amount of industrial, domestic and agricultural waste water. In addition, 
Jihongtan Reservoir is located in suburb area, rainfall and dusts falling on the reservoir contain lesser nutrients. Moreover, with the help of the Water Diversion Project from Yellow River to Qingdao in Shandong which will supply steady stream of water into Jihongtan Reservoir, the exchange rate of the water is high with water contently maintain a flowing state, thus leads to reduction of deposition of nutrition salts. Due to these reasons, trophic materials and organic pollutants in the reservoir are low, the growth of algae and phytoplankton in the water are limited, hence reduced the chlorophyll a concentration in the reservoir area.

Comparison result. Though Laoshan Reservoir lays in mountainous areas, with input from numerous rives which comes from the region with agricultural, tourism and industrial related waste water, the trophic state index in Laoshan Reservoir overall is higher than the average trophic state index for lakes and reservoirs in East Shandong Peninsula area. On the other hand, though Jihongtan Reservoir is a plain reservoir, it is a dam type plain reservoir with closed circumference, which mainly accepts water input from the Water Diversion Project from Yellow River to Qingdao in Shandong. As such, there is less significant influence of industrial, domestic and agricultural waste water on Jihongtan Reservoir. Water exchange rate in the reservoir is relatively high. Thus, comprehensive nutrient states index for Jihongtan Reservoir is generally lower than the average index for East Shandong Peninsula Lakes and Reservoirs area. Therefore, compare trophic level of these two reservoirs, trophic state of Jihongtan Reservoir is better than that of Laoshan Reservoir; the latter has already been facing the problem of eutrophication and awaiting improvements.

Comparison of Trophic States for Typical Lakes and Reservoirs of Different Regions. For lakes and reservoirs of different regions, we chose Nansi Lake of Yishusi River Lakes and Reservoirs area and Dongping Lake of Yellow River Basin Lakes and Reservoirs area. Nansi Lake belongs to mixed type lakes and Dongping Lake belongs to plain farmland type lakes, thus they have a strong comparability for lakes and reservoirs of different regions.

Nansi Lake. Nansi Lake is the largest freshwater lake in Shandong. It is made up of four connected lakes, from South to North - Nanyang Lake, Dushan Lake, Shaoyang Lake and Weishan Lake. Surface area for Nansi Lake is $1266 \mathrm{~km}^{2}$ and its average depth is $1.46 \mathrm{~m}$ which makes the lake an important transferring channel and regulation and storage hub for the East Ling of the Water Diversion Project from Southern China to Northern China. Nansi Lake belongs to the Haihe River Basin, Si River system. It accepts water input from Shandong, Henan, Anhui and Jiangsu provinces with 53 rivers entering the lake and a storage capacity of $17.02 \times 10^{8} \mathrm{~m}^{3}[14]$.

Nansi Lake River Basin is comprised of mountains, hills and piedmont plain in the east part of the region, as well as west to east Yellow River Floodplain in the west. Topography and land usage in the region is complex, which leads to a wide spread of industrial, agricultural and domestic pollution sources all over the place. Various types of pollutant enter Nansi Lake via flow of rivers entering the lake[15]. According to statistics collected in 2009 and 2010, trophic states indexes for the lake are 49.6 (medium level) and 50.77 (slightly eutrophication level). By observing data from 2009 to 2011, it is obvious that Ammonia concentration and total phosphorous concentration in Nansi Lake both reached eutrophication level, Secchi Dish Depth was only around $0.5 \mathrm{~m}$, potassium permanganate index was at medium trophic level and concentration of chlorophyll a was low in 2009 and 2010 , both under $4 \mathrm{mg} / \mathrm{m}^{3}$, but reached $17.5 \mathrm{mg} / \mathrm{m}^{3}$ in 2011 .

The surrounding area of Nansi Lake has been one of China's major cotton and grain production base. Mineral resources in the region are relatively abundant which, over the years has lead to the establishment of quite a large number of coal, copper and rare earth mineral extraction bases, driven the development of a range of industrial sector such as electrical, mechanical, chemical and food, etc. As a result, well-developed industrial and agricultural sector leads to large amount of industrial and domestic waste water as well as leaching water rich of fertilizer and pesticides from agricultural activities flow into the lake. This greatly increased the content of trophic material and organic matter in the lake causing severe pollution on the water quality of Nansi Lake. Moreover, Nansi Lake itself has been the largest freshwater fishery base in Shandong, feeding materials released by 
human and fish excreta also add a large quantity of trophic materials such as nitrogen and phosphorus which speed up the eutrophication process in the lake.

Nansi Lake is a typical shallow lake. Average depth in the lake is less than $1.5 \mathrm{~m}$. Nansi Lake is with slow flow rate and long water cycling period. Water exchange cycle in the lake is longer than 503 days. Dilution and degradation ability of the lake is low, environment capacity is small and pollution of water in the lake has been severe[16]. Furthermore, the increase of phytoplankton in water and floating of loosely suspended sediments from soil all lowered the Secchi Dish Depth of the water in Nansi Lake.

Dongping Lake in Taian. Donping Lake is the second largest freshwater lake in Shandong, with a total area of $627 \mathrm{~km}^{2}$ and perennial storage capacity of $1.5 \times 10^{8} \mathrm{~m}^{3}$. The lake locates in the junction of three counties - Dongping, Liangshan and Wenshang. With Liangji Canal on the west, Yellow River on the south, and Dawen River on the east, the lake has been a regulating reservoir accepting and treating floods of large scale from downstream of Yellow River and Dawen River.

According to data collected in 2009 and 2010, trophic states index of Dongping Lake was 60.9 (moderately eutrophic) and 49.61 (medium trophic) respectively. By observing date from 2009 to 2011, it is clear that ammonia concentration of Dongping Lake was in mild eutrophication condition; total phosphorus concentration and potassium permanganate index were lower than those of Nansi Lake, at medium trophic level; average Secchi Dish Depth of the lake was $0.98 \mathrm{~m}$, which is much than Nansi Lake; content of chlorophyll a was relatively high.

The mainly water supply source of Dongping Lake comes from Dawen River thus water quality of Dongping Lake largely depends on that of rivers in Dawen River Basin. As coastal areas along Dawen River Economic Development, especially in terms of coal mines and paper mills along the river, large quantity of industrial waste water flow into the river. This leads to a certain amount of pollution in Dawen River and increased the content of trophic material and organic matter in Dongping Lake.

Dongping Lake belongs to plain farmland lakes and reservoirs with chemical fertilizers and pesticides been the major polluting source for the lake. There are large quantity of nitrogen and phosphorus in chemical fertilizers and pesticides, which been carried by rives and surface runoff, gradually accumulate in lakes, adding up to the eutrophication process in lakes and reservoirs. In addition, part of the industrial and residential waste water around the lake also took a part in increasing the amount of trophic material and organic matter in the lakes and reservoirs. As a result of sufficient nutrients and light which create a good growing environment for algae, a huge number of algae and phytoplankton lead to an increase in concentration of chlorophyll a in the lake.

Comparison result. From the comparison results from trophic state analysis above, trophic state of Dongping Lake in Yellow River Basin Lakes and Reservoirs area is generally better than that of Nansi Lake in Yishusi Lakes and Reservoirs area of Huai River Basin. The main reason for this is the complexity in terms of topography and land usage conditions in Nansi Lake. Large quantity of trophic materials such as nitrogen and phosphorus from industrial, residential, farming and freshwater aquaculture are brought into the lake. Furthermore, Nansi Lake is a typical shallow lake with slow flow rate and long cycling period. Therefore, among the 26 nation controlled lakes and reservoirs in China, Nansi Lake is a mildly eutrophic lake which needs stronger control of eutrophication and improvement in water quality.

\section{Controlling and Improving Measure for Eutrophication of Lakes and Reservoirs}

Eutrophication of lakes and reservoirs seriously hampered sustainable soci- economic development. With regard to the current pollution state of lakes and reservoirs in Shandong, after comparison analysis between different lakes and reservoirs, we come up with the following controlling and improving measure.

Laoshan Reservoir. Laoshan Reservoir has well developed tourism sector thus it is essential to control the release of waste water from surrounding residential areas. To prevent eutrophication 
process in lakes and reservoirs, it is a must to strengthen the government's capacity to intervene, build more complete regulating system of lakes and reservoirs so as to make sure that relevant laws and regulating can be implemented effectively. More sewage treatment facilities should be built around the lakes and reservoirs. At the same time, more effort should be put in to advocate environmental protecting knowledge and regulations, so as to raise the awareness of public on environmental protection, thus ensure the public take an active part in protecting the environment around lakes and reservoirs.

Jihongtan Reservoir. For Jihongtan Reservoir, preventing measure should be taken as the main method. Before carrying out the diversion project, water source needs to be monitored. As a closed reservoir, as long as quality of source water is ensured, eutrophication of water will be controlled.

Nansi Lake. Nansi Lake has a wide source of waste materials and severe siltation problem thus a comprehensive managing measure should be applied. First of all, it is essential to control the input of waste materials. The industrial sector should gradually phase out industrial enterprise of high pollution, high energy usage and high water wastage so as to achieve a Cleaner Production. The agricultural sector needs to put in more effort developing ecological agriculture, control usage of fertilizer and pesticides to a reasonable level, control soil erosion condition in upper stream region and gradually form an eco-cycle model of economic development in terms of farming and freshwater aquaculture to reduce trophic materials in water. In the residential sector, it is important to promote the use of phosphorus free or low phosphorus synthetic detergents among people as well as build up more sewage treatment facilities around the lakes and reservoirs. By sediment dredging, aeration of water body, introduction of high quality water source, reduction of endogenous contamination and acceleration of water cycling speed to improve the water quality. Besides, restore the wetland ecosystem is also a necessary measure to prevent and control eutrophication in lakes and reservoirs.

Dongping Lake. The eutrophication in Dongping Lake largely depends on water quality of Dawen River. Hence treatment on Dawen River is an important way to prevent further aggravation of eutrophication in Dongping Lake. Besides control the input of lake pollutants along the river, there should also be tighter management on coal mines and paper mills on the coastal area of Dawen River, require their wastewater discharged to reach standards and make full use of sewage treatment plants.

\section{Acknowledgements}

This Paper is supported by Natural Science Foundation of Shandong Province (NO. ZR2009BL007)

\section{References}

[1] Wu Yu. Eutrophication: The Difficult Problems of lakes treating in China [J]. Ecological Economy, 2008, (9):14-19

[2] Ma Jing-an, Li Hong-qing. Preliminary Discussion on Eutrophication Status of Lakes, Reservoirs and Rivers in China and Overseas [J]. Resources and Environment in the Yangtze Basin, 2002, 11(6):575-577

[3] Yang Guishan, Ma Ronghua, Zhang Lu, et al. Lake Status, Major Problems and Protection Strategy in China[J]. Journal of Lake Sciences, 2010, 22(6):799-810

[4] Song Yandong. Environmental Quality Report in Shandong Province [R]. Shandong Environmental Protection Department, 2011.5:237-238

[5] Zhu Minghan. 2006 Water Resources Overview [EB/OL]. http://www.sdwr.gov.cn/sdsl/pub/cms/2092/283/294/14718.html.2008.10.15 
[6] Yu Ai-min, Shang Guangping, Yu Yang. Discussion and Case Study Overall Evaluation Method of Lake Eutrophication [J]. Inner Mongolian Environmental Sciences, 2009, 21(3):52-55

[7] Wang Ming cui, Liu Xueqin Zhang Jianhui. Evaluate method and classification standard on lake eutrophication [J]. Environmental Monitoring In China, 2002, 18(5):47-50

[8] James M. Omernik. Perspectives on the Nature and Definition of Ecological Regions [J]. Environmental Management, 2004, 34 (1):27-38

[9] Cao Zhengmei, Lang Yinhai, Liu Rong. Investigation and Assessment of Eutrophication Level for Laoshan Reservoir [J]. Transactions of Oceanology and Limnology, 2009(2):103-107

[10] Schindler DW. Recent advances in the understanding and management of eutrophication [J]. Limnology and Oceanography, 2006, 51(1, Part 2): 356-363

[11] Reynolds CS, Irish AE, Eliott JA. The ecological basis for stimulating phytoplankton responses to environmental change [J]. Ecol Modeling, 2001, 140:271-291

[12]Zhang Yunlin, Qin Boqiang, Chen Weimin, et al. Distribution, Seasonal Variation and Correlation Analysis of the Transparency in Taihu Lake [J]. Transactions of Oceanology and Limnology, 2003, (2):30-36

[13] hao Xian-Fu, Yu Jun, Ge Jian-Hua, et al. Study on the Phytoplankton and Water Quality of Jihongtan Reservoir in Qingdao [J]. Acta Hydrobiologica Sinica, 2005, 29(6):639-644

[14] Shen Jie, Zhang Zu-lu, Yang Li-yuan, et al. Nansi Lake - Environment and Resource Development [M]. Beijing: Seismological Press, 2008:1

[15] Wu Zhou-hu, Mu Jin-bo, Xie Gang, et al. Analysis of Water Environmental Quality Variation Trends in Nansi Lake and Its Joined Rivers[J]. Research of Environmental Sciences, 2010, 23(9):1167-1173

[16]Zhang Zu-lu, Liang Chun-ling, Guan Yan-bo. Assessment of Wetland Ecosystem Health in the Nansi Lake [J]. China Population Resources and Environment, 2008, 18(1), 180-184 